\title{
Automatic Segmentation of Lymph Vessel Wall using Optimal Surface Graph Cut and Hidden Markov Models
}

\author{
Jonathan-Lee Jones, Ehab Essa, and Xianghua Xie
}

\begin{abstract}
We present a novel method to segment the lymph vessel wall in confocal microscopy images using Optimal Surface Segmentation (OSS) and hidden Markov Models (HMM). OSS is used to preform a pre-segmentation on the images, to act as the initial state for the HMM. We utilize a steerable filter to determine edge based filters for both of these segmentations, and use these features to build Gaussian probability distributions for both the vessel walls and the background. From this we infer the emission probability for the HMM, and the transmission probability is learned using a Baum-Welch algorithm. We transform the segmentation problem into one of cost minimization, with each node in the graph corresponding to one state, and the weight for each node being defined using its emission probability. We define the inter-relations between neighboring nodes using the transmission probability. Having constructed the problem, it is solved using the Viterbi algorithm, allowing the vessel to be reconstructed. The optimal solution can be found in polynomial time. We present qualitative and quantitative analysis to show the performance of the proposed method.
\end{abstract}

\section{INTRODUCTION}

The lymphatic system is vital for maintaining healthy functionality in the body; fulfilling this task by the transport of fluids and immunological cells from and to the blood and interstitial spaces. The lymphatic system at its most basic is a system of pipes and valves to ensure unidirectional flow. The vessels are thin walled and not under pressure, so without the valves it would be impossible to maintain unidirectional flow.

Confocal microscopy is a method that allows for the visualization and imaging of $3 \mathrm{D}$ volume objects that are thicker than the focal plane of a conventional microscope using a point detector, situated in front of the photo-detector. This serves to attenuate any signals originating outside the focal region, allowing for "optical slices" to be obtained through a thick object and stored in a computer [1]. This optical sectioning is much stronger than the depth of focus of the microscope; allowing for the objects in the layer of interest to be in focus, and making the capturing of the relevant image to the computer trivial. Unlike conventional microscopy only one point of the sample is illuminated at a time in confocal microscopy. During operation, a laser beam scans the object pixel by pixel, line by line and any light outside of the focal plane is blocked by the point detector.

\footnotetext{
Department of Computer Science, Swansea University, Singleton Park, Swansea, UK. Correspondence: x.xie@swansea.ac.uk http://csvision.swan.ac.uk

The project was funded by NISCHR BRU-AMIV. J. Jones is funded by EPSRC as a PhD student. E. Essa was funded by Swansea University as a PhD student and NISCHR as a postdoc, and he is also affiliated with Mansoura University, Egypt.
}

This data is then reconstructed by the computer into the 3D image $I(x, y, z)$. As only one point in the sample is illuminated at a time, building up an image requires scanning the object over a regular raster (i.e. a rectangular pattern of parallel scanning lines). The thickness of the focal plane is defined by the inverse of the square of the numerical aperture of the objective lens, but can also affected by the optical properties of the specimen and the ambient index of refraction. Figure 1 shows an example image of a lymph vessel obtained using a confocal microscope. There are many examples of 2D segmentation carried out on confocal images, such as [27] but in our method we propose using object tracking to provide a method to segment the $3 \mathrm{D}$ volume. Object tracking is becoming a useful tool in the medical field [7], [8], [9], as well as its traditional use in a variety of different applications[5], [6], [2], [3], [4]. The object can be represented in various ways (points, geometric shape, and contours for example). Some, such as geometric shapes, are more suitable for representing rigid objects; whereas tracking the object contour can be used to segment a complex and nonrigid object.

In medical imaging, identifying 3D surfaces is of utmost importance. It is often the case that this is done by using a 2D method, as a great deal of work has been carried out in this area [12], [11], [13], [14], [15], [16] but when these methods are extended to segment a 3D volume, usually by looking at a series of slices, contextual information between the layers that make up the volume is often lost. There have been attempts to extend these methods into 3D [17] but these have often proved to be computationally expensive. Another problem with utilizing 2D methods is that they can produce local minima. One method for 3D segmentation that allows for a global minimum is Optimal Surface Segmentation(OSS) [10]. OSS provides a polynomial time method for simultaneously providing a global segmentation of a 3D volume with minimal or no user intervention, and in our method forms the basis of determining the initial segmentation, used to initialize the HMM.

The Hidden Markov Model (HMM) is a stochastic model, in which over a finite set of hidden states, the Markov property can be assumed to be satisfied. HMMs have been utilize in a great variety of fields, such as speech recognition ([20], [19]) , classification ([18], [5]) and tracking ([21], [22]). HMM has been used in the medical field both for segmentation ([5], [23])and image processing ([24]). In this paper, we propose multi-border segmentation and tracking method based on the HMM, in order to delineate the inner and outer vessel borders in lymphatic images with the 


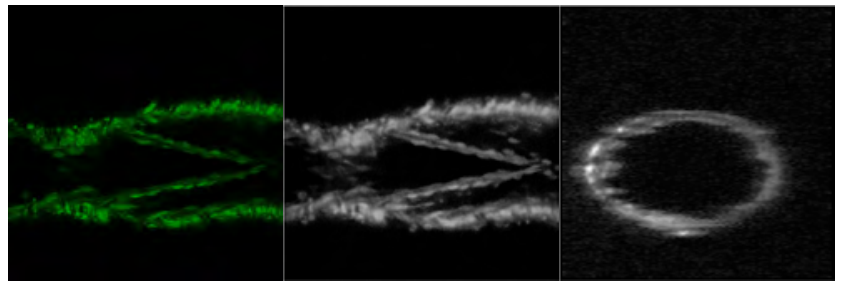

Fig. 1. Image on far left shows original image, the center after VED has been applied, and the image on the right shows the image after transformation

presence of noise and occlusions.

\section{PROPOSED METHOD}

In our proposed method, we first apply preprocessing steps to the image. These involve applying Vessel Enhancing Diffusion (VED) to the image to reduce the noise and help lessen the detrimental effect of the occluded areas. VED has been shown to improve segmentation results of vessel structures ([25]). The images were then transformed to cross sections, aligned and scaled to uniform dimensions to aid the training. The stages of pre-processing are shown in Figure 1. Once the images were prepared, OSS was carried out, to be used as the initial segmentation for the HMM segmentation stage.

\section{A. Graph Construction}

The volumetric image we wish to segment, now comprises of a series of slices through the lymph vessel. In order to find the optimal surface, we transform these from Cartesian to Polar coordinates. Each slice representing the $(\mathbf{x}, \mathbf{z})$ plane, and position of that slice in the stack gives the $\mathbf{y}$ axis, as if looking at a topographical landscape feature. Our image can therefore be represented as a 3D matrix $\mathcal{I}(x, y, z)$. The size in any given dimension can be represented as $X, Y$ and $Z$. The surface can thusly be defined:

$$
\begin{array}{r}
\mathcal{I}:(x, y) \rightarrow \mathcal{I}(x, y), \text { where }: \\
x \in \mathbf{x}=\{0, \ldots, X-1\}, \\
y \in \mathbf{y}=\{0, \ldots, Y-1\}, \text { and } \\
\mathcal{I}(x, y) \in \mathbf{z}=\{0, \ldots, Z-1\}
\end{array}
$$

In this way, any given surface $\mathcal{I}$ intersects with exactly one voxel of each column parallel to the $\mathbf{z}$-axis. We can therefore construct a node-weighted graph $\mathcal{G}=(\mathcal{V}, \mathcal{E})$ can be constructed for $\mathcal{I}$. In the graph $\mathcal{G}$ each voxel $v$ is connected to column-wise with its bottom neighbor, with its neighboring voxels in the subsequent layer (with $\Delta$ deflection), and with neighboring columns (with $\delta$ deflection) with $\infty$ cost; the final voxel in each column has a bidirectional arc to its neighbor.

\section{B. Cost Term}

For every node $\mathcal{V}(x, y, z) \in \mathcal{V}$ in the graph represents one voxel in $\mathcal{I}(x, y, z) \in \mathcal{I}$ with a given weight $w(x, y, z)$. This weight, $w$, can be derived from a steerable edge based filter applied to the polar transformed image. The steerable filter is a linear combination of differentially orientated instances of a base filter. A set of $n$ order derivatives of Gaussian filters $G_{n}(x, y)$ in different orientations are used to highlight edge features along a border. These can be defined as follows:

$$
\mathcal{G}_{n}^{\theta}(x, y)=\sum_{j=1}^{M} k_{j}(\theta) \mathcal{G}_{n}^{\theta}(x, y)
$$

where $\mathcal{G}_{n}^{\theta}(x, y)$ is the rotated version of $\mathcal{G}_{n}(x, y)$ at $\theta$ orientation, and $k_{j}, 1 \leq j \leq M$ are interpolation functions. Due to the derivatives in the direction being invariant, regardless of rotation, steerable filters are effective at highlighting orientated structures, in this case the edges, than other filter types, especially in situations such as in the lymph images where there is a lot of noise [26]. The cost of any voxel $v$ can be given as $w_{v}=g(v)$ where $g$ is the edge based cost derived from the steerable filter. The total energy cost $\mathcal{E}$ for every voxel in the $s-t$ cut can therefore be represented by:

$$
\mathcal{E}=\sum_{v \in V} w(v)
$$

\section{HMM Segmentation}

The results from the OSS are used to set the initial state of our HMM. We trained the HMM with 1024 image slices from 2 confocal samples, using the unraveled polar coordinates for the voxels in our image. It is computationally very expensive to track all the border points in the polar coordinates, but in our case, this is unnecessary. We used an RBF to approximate the border of interest, with the hidden states of the HMM referring to the RBF centers. The border was evenly sampled into $M$ points and from each of these a line segment is extended either side, perpendicular to the border's tangent at that point, with line segments each having $K$ points, with the index of the RBF centers denoted $\phi=1, \ldots, M$ and the index along each of these normal lines being denotes as $\psi=1, \ldots, K$ where $K$ is an odd integer. Our initial RBF centers are derived from the result of the OSS, and are located at the center of the normal line $\psi=$ $(K+1) / 2$. The steerable filter is used to determine the edge, in this case the vessel wall, and the emission probability is computed using Gaussian distributions calculated from the image observation. Our transmission probability is learned from the training set. In our proposed HMM, we denote all possible sequences of hidden states as $\mathcal{Q}=\{q\}$ where $q=\left\{q_{1}, \ldots, q_{\phi}, \ldots, q_{M}\right\}$ is one of the possible state sequences (corresponding to possible RBF center locations) and $q_{\phi}$ is the state being on the normal at $\phi$. The observations $O$ of the HMM are extracted from the normal lines as such that $O=\left\{o_{1}, \ldots, o_{\phi}, \ldots, o_{M}\right\}$, and the HMM is specified by the probability measures $\lambda=(A, B, \pi)$. Let $O_{\phi}=$ $\left\{o_{\phi, 1}, \ldots, o_{\phi, \psi}, \ldots, o_{\phi, K}\right\}$ be the set of features along the normal $\phi$ and $o_{\phi, \psi}$ be a feature extracted from point $\psi$ on the line, thusly $P\left(o_{\phi, \psi} \mid F G\right)$ and $P\left(o_{\phi, \psi} \mid B G\right)$ are the probabilities of the feature being foreground or background respectively. The state-emission probability can therefore be 

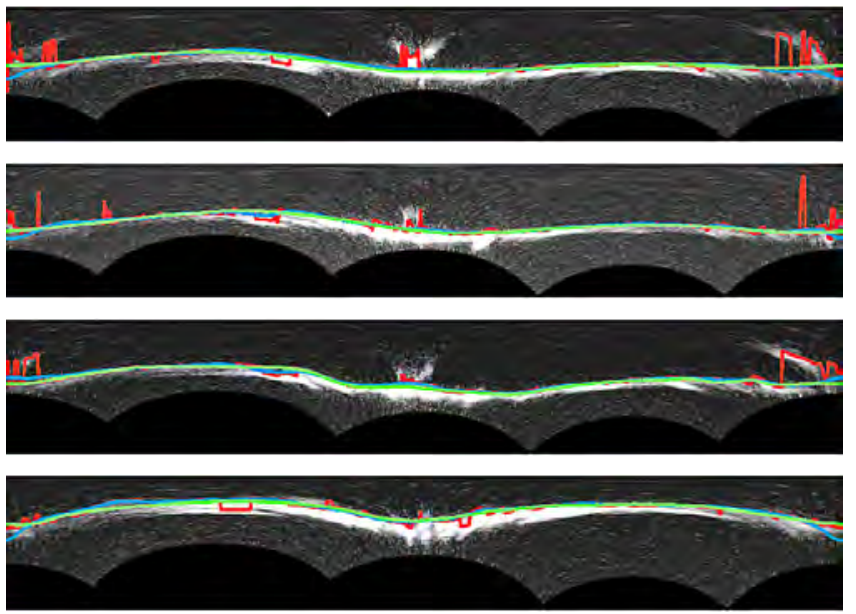

Fig. 2. Results for the inner wall of the lymph vessel. Light blue is the proposed method, red OSS only and green the ground truth

defined as:

$$
P\left(O_{\phi} \mid q_{\phi}\right) \propto P\left(o_{\phi, \psi} \mid F G\right) \prod_{\psi \neq q_{\phi}} P\left(o_{\phi, \psi} \mid B G\right)
$$

We then use the Viterbi algorithm, which is a refinement of the Baum-Welch algorithm. The Baum-Welch algorithm uses Expectation-Maximization method to compute the maximum likelihood in the HMM the probability of moving from a state $i$ at normal $\phi$ to new state $j$ at normal $\phi+1$, where $1 \leq \phi \leq M$ and $1 \leq i, j \leq K$ between states $q$ with two normals $\phi$ and $\phi+1$ as:

$$
\xi_{\phi}(i, j)=P\left(q_{\phi}=i, q_{\phi+1}=j \mid O, \lambda\right)
$$

In the Viterbi algorithm, the sequence of states $q *$ can be efficiently found given the image observation $O_{t}$ and the HMM model $\lambda$ :

$$
q *=\underset{q \in Q}{\operatorname{argmax}} P\left(q \mid O_{t}, \lambda\right) .
$$

\section{RESULTS}

The results were drawn from 3,072 slices from 6 different experiments, separate from those used in the training set. Figure 2 and Figure 3 show the results obtained for segmentation of the inner and outer walls respectively, before conversion back from polar to Cartesian coordinates. The results show the improvement of the HMM method (blue) over the OSS alone (red). The results for the outer wall are significantly better than those for the inner. In both methods (though less so in the proposed method), this is because the valve extends from the wall, into the lumen, this makes it hard to even manually label the slices where the valve originates. Our proposed method shows a significant improvement in these regions, as well as those with very poor contrast or missing areas. because the training helps the segmentation to cope with interference and gaps in the edge.

Table I provides a quantitative comparison between the proposed method and the results obtained using OSS alone.
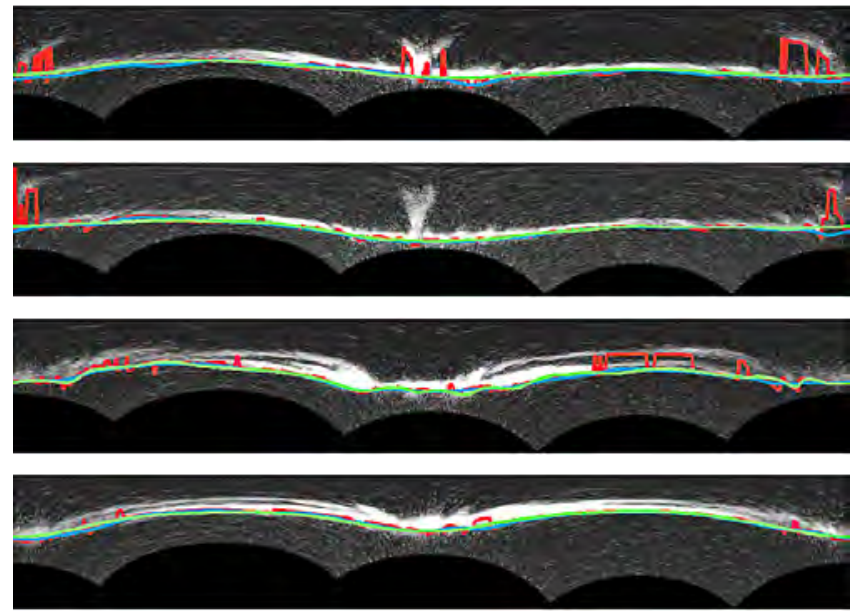

Fig. 3. Results for the outer wall of the lymph vessel. Light blue is the proposed method, red OSS only and green the ground truth

The proposed method clearly benefited from HMM modeling and produced consistently better results.

In Figure 4, we show some typical results of segmentation of both inner and outer walls of the vessel using the proposed method. It can be seen that by combining optimal surface segmentation with HMM, a coherent and smooth segmentation of both boundaries is achieved in the presence of noise and frequent weak edges.

\section{CONCLUSIONS}

Our results show that our proposed method is a reliable means to automatically segment bother inner and outer surfaces of the walls of the lymph vessel, allowing for it to be visualized in 3D. By using OSS and HMM, we are able to find a global minimum to the segmentation problem in polynomial time without user intervention despite the low quality, and diffuse nature of the confocal images taken from the lymph vessels.

\section{ACKNOWLEDGEMENTS}

The authors would like to thank David Zawieja and Roul van Loon for providing the imaging data.

\section{REFERENCES}

[1] J.B. Pawley (Editor), Handbook of Biological Confocal Microscopy, New York: Plenum, 1995.

[2] Aljuaid, H. and Mohamad, D. Object tracking simulates babysitter vision robot using GMM, In: SoCPaR; Hanoi, December 2013. pp. 60-65, IEEE

[3] Beymer, D., Konolige, K.: Real-time tracking of multiple people using continuous detection. In: ICCV Frame-Rate Workshop (1999)

[4] Xie, L., Zhu, G., Wang, Y., Xu, H., Zhang, Z.: Real-time vehicles tracking based on kalman filter in a video-based ITS. In: ICCCAS (Volume 2). May 2005; IEEE

[5] Mansor, S., Noble, J.: Local wall motion classifcation of stress echocardiography using a hidden markov model approach. In: ISBI, 2008

[6] Mondal, A., Ghosh, S., Ghosh, A. Efficient silhouette based contour tracking. In: ICACCI, 2013. pp. 1781-1786. IEEE

[7] Abolmaesumi, P., Sirouspour, M., Salcudean, S.: Real-time extraction of carotid artery contours from ultrasound images. In: CBMS 2000

[8] Cheng, J., Foo, S., Krishnan, S.M.: Watershed-presegmented snake for boundary detection and tracking of left ventricle in echocardiographic images. IEEE T-ITB 10(2),pp. 414-416 (2006) 

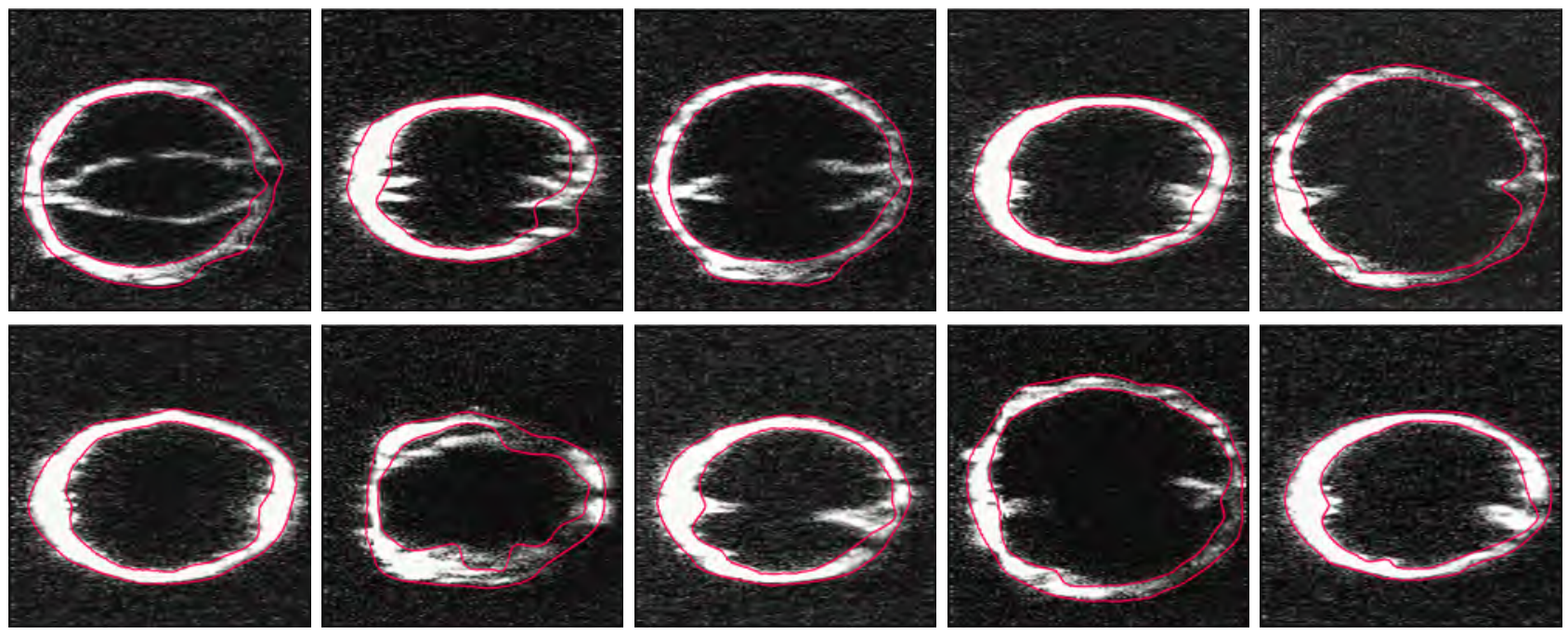

Fig. 4. Results showing the segmented walls (red) on the wrapped images.

TABLE I

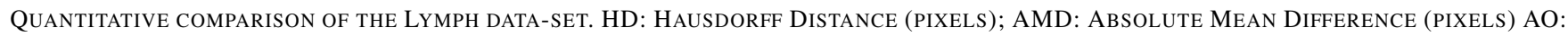
Area Overlap(\%); Spec: Specificity (\%); Sens: Sensitivity (\%). Bold font indicates best Performance.

\begin{tabular}{|l|c|c|c|c|c|c|}
\hline Method & & HD & AMD & Spec & Sens & AO \\
\hline \hline \multirow{2}{*}{ Outer Wall -OSS } & Mean & 51.0330 & 7.0631 & 97.34 & 91.15 & 90.06 \\
& STD & 22.59 & 3.55 & 0.62 & 5.56 & 5.63 \\
\hline \multirow{2}{*}{ Outer Wall -Proposed method } & Mean & $\mathbf{2 3 . 3 1}$ & $\mathbf{4 . 6 7}$ & $\mathbf{9 9 . 1 6}$ & $\mathbf{9 4 . 8 0}$ & $\mathbf{9 3 . 2 9}$ \\
& STD & 2.88 & 10.14 & 0.69 & 4.6 & 4.81 \\
\hline \multirow{2}{*}{ Inner Wall -OSS } & Mean & 55.52 & 7.06 & 96.18 & 92.73 & 91.71 \\
& STD & 3.17 & 13.57 & 0.81 & 4.28 & 4.07 \\
\hline \multirow{2}{*}{ Inner Wall -Proposed method } & Mean & $\mathbf{1 3 . 7 2}$ & $\mathbf{3 . 3 1}$ & $\mathbf{9 8 . 4 9}$ & $\mathbf{9 8 . 0 0}$ & $\mathbf{9 6 . 1 1}$ \\
& STD & 5.35 & 9.16 & 3.88 & 5.37 & 7.67 \\
\hline
\end{tabular}

[9] Behrens, T., Rohr, K., Stiehl, H.S. Robust segmentation of tubular structures in 3-D medical images by parametric object detection and tracking. Systems, Man, and Cybernetics, Part B: IEEE Trans. Cybern. (Volume:33, Issue: 4 ) pp. 554 - 561. August 2003, IEEE.

[10] Li K., Wu X., Chen, D.Z., Sonka, M. Optimal Surface Segmentation in Volumetric Images-A Graph-Theoretic Approach. TPAMI, (Volume:28 , Issue: 1 ) pp. 119-134. January 2006. IEEE

[11] J.-L. Jones, X. Xie, and E. Essa, Combining Region-based and Imprecise Boundary-based Cues for Interactive Medical Image Segmentation, Int J Numer Method Biomed Eng, volume 30, issue 12, pp. 16491666, December, 2014

[12] J.-L. Jones, E. Essa, X. Xie and J. Cotton, Interactive segmentation of lumen border in OCT, In Proc. CMBE, December 2013.

[13] E. Essa, X. Xie, I. Sazonov, P. Nithiarasu, D. Smith, Shape Prior Model for Media-Adventitia Border Segmentation in IVUS using Graph Cut, In MICCAI Medical Computer Vision, September 2012.

[14] A.X. Falco, J.K. Udupa, F.K. Miyazawa, An Ultra-Fast User-Steered Image Segmentation Paradigm: Live Wire on the Fly, IEEE Trans. Med. Imag., vol. 19, Jan.,2000

[15] M. Sonka, X. Zhang, S. DeJong, S. Collins, C. McKay, Segmentation of Intravascular Ultrasound Images: A Knowledge-Based Approach, IEEE Trans. Med. Imag., vol. 14, pp.719-732, Aug., 1995

[16] A. Schenk, G. Prause, H.-O. Peitgen, Local Cost Computation for Efficient Segmentation of 3D Objects with Live Wire, Proc. SPIE Int'l Symp. Medical Imaging: Image Processing, vol. 4322, pp.1357-1364, 2001

[17] A.X. Falco, J.K. Udupa, A 3D Generalization of User-Steered LiveWire Segmentation, Med Image Anal, vol. 4, pp.389-402, Dec., 2000

[18] A. Lacey, J. Deng, and X. Xie, Protein Classification using Hidden Markov Models and Randomised Decision Trees, In Proc. BMEI, pp. 659-664, October 2014.
[19] Juang, B.-H.,On the hidden Markov model and dynamic time warping for speech recognition A unified view. AT\&T Bell Laboratories Technical Journal (Volume:63, Issue: 7) pp. 1213 - 1243, September 1984, Alcatel-Lucent.

[20] Rabiner, L.: A tutorial on hidden markov models and selected applications in speech recognition. Proceedings of the IEEE 77(2),pp. 257-286 (1989)

[21] Chen, Y., Rui, Y., Huang, T.: JPDAF based HMM for real-time contour tracking. In: CVPR (2001)

[22] Chen, Y., Rui, Y., Huang, T.: Multicue HMM-UKF for real-time contour tracking. IEEE PAMI 28(9),pp. 1525-1529 (2006)

[23] AlZubi, S., Islam, N., Abbod, M. Enhanced Hidden Markov Models for accelerating medical volumes segmentation. In: GCC Conference and Exhibition, pages 287 - 290. Dubai, 2011 IEEE

[24] Jixiang Z., Xiangling Z., Zhijun P., Medical Image Denoising Using Hierarchical Hidden Markov Model in the Wavelet Domain. In: ECTS Workshop (Volume:2), pp. 857-860. Hubei, March 2009. IEEE.

[25] Manniesing R., Viergever M.A., Niessen W.J., Vessel enchancing diffusion. Med Image Anal, Volume 10, Issue 6, pp. 815 - 825 June 2006.

[26] Freeman W. T., Adelson E. H., The design and use of steerable filters, TPAMI, vol. 13, no. 9, pp. 891906, 1991, IEEE.

[27] Meziou, L.; Histace, A.; Precioso, F.; Matuszewski, B. J.; Murphy, M. F. Confocal microscopy segmentation using active contour based on alpha $(\alpha)$-divergence. ICIP pp. 3077 - 3080, 2011 